\title{
Increased prevalence of low oligomeric state surfactant protein $D$ with restricted lectin activity in bronchoalveolar lavage fluid from preterm infants
}

\author{
Sailesh Kotecha, ${ }^{1}$ Philip L Davies, ${ }^{1,2}$ Howard W Clark, $^{3}$ Eamon P McGreal ${ }^{1}$
}

\begin{abstract}
- Additional material is published online only. To view please visit the journal online (http://dx.doi.org/10.1136/ thoraxjnl-2012-202729).

1 Department of Child Health, Institute of Molecular and Experimental Medicine, Cardiff University School of Medicine, Cardiff, UK

${ }^{2}$ Royal Hospital for Sick

Children, Yorkhill, Glasgow, UK (current address)

${ }^{3}$ Department of Child Health, NIHR Respiratory Biomedical Research Unit, Clinical and Experimental Sciences, Faculty of Medicine, University of Southampton, Southampton, UK
\end{abstract}

\section{Correspondence to} Dr Eamon P McGreal, Department of Child Health, Institute of Molecular and Experimental Medicine, Cardiff University School of Medicine, Heath Park, Cardiff CF14 4XN, UK; mcgrealep@cf.ac.uk

Received 13 September 2012 Revised 14 December 2012 Accepted 11 January 2013 Published Online First 6 February 2013
To cite: Kotecha $S$, Davies PL, Clark HW, et al. Thorax 2013;68:460-467.

\begin{abstract}
Background Surfactant protein D (SP-D) is a soluble oligomeric C-type lectin known to protect against lipopolysaccharide and ventilator-induced lung injury in preterm lambs. Here we assess the expression and functional status of SP-D in bronchoalveolar lavage fluid (BALF) from preterm infants at risk of chronic lung disease (CLD) of prematurity and term controls. This is the first systematic evaluation of SP-D function in any clinical cohort.

Methods SP-D was quantified in BALF from 28 ventilated preterm infants and five ventilated term infants. SP-D lectin activity was tested in a zymosan binding assay followed by sodium dodecyl sulfate polyacrylamide gel electrophoresis (SDS-PAGE) and western blot in BALF from the same infants. SP-D lectin activity was also tested towards maltose-agarose and mannan for selected BALF samples.
\end{abstract}

Results SP-D expression was lower on day 1 in those preterm infants who subsequently developed CLD but increased over the first 5 days of life in term and preterm neonates. The percentage of neonatal SP-D capable of binding zymosan rarely exceeded $50 \%$ in any BALF sample and was 3.5 times lower in preterm infants than term infants on day 1 of life. Similar binding defects were observed towards maltose-agarose and mannan. SDS-PAGE analysis revealed that zymosan-bound SP-D was more highly oligomerised ( $\geq 12$-mers) than unbound SP-D, which was composed primarily of trimers and lower oligomeric forms.

Conclusions Substantial and functionally relevant variation in the expression and oligomeric distribution of SP-D exists between preterm and term neonatal lung secretions. This has implications for proposed SP-D replacement therapy in this population.

\section{INTRODUCTION}

Infectious and inflammatory disease is a particular problem for premature infants due to recognised deficiencies of innate and adaptive immunity ${ }^{12}$ Many very premature infants develop respiratory distress syndrome (RDS) due to pulmonary immaturity and this, combined with infection and ventilator-induced injury, contributes to the development of chronic lung disease (CLD) of prematurity or bronchopulmonary dysplasia. ${ }^{3}$

Surfactant protein D (SP-D) is a soluble C-type lectin best described in the lung but also expressed at other mucosal sites. Like other soluble C-type lectins including mannose binding lectin and surfactant protein A (SP-A), the lectin domain of SP-D

\section{Key messages}

What is the key question?

- Is there a quantitative or functional deficiency of the antimicrobial and inflammatory regulator, surfactant protein-D (SP-D), in lung fluid from preterm infants at risk of chronic lung disease of prematurity?

\section{What is the bottom line?}

- Substantial and significant variation in the expression, oligomeric state and lectin activity of SP-D is evident in preterm infants compared with term infants and also in preterm infants at risk of developing chronic lung disease.

\section{Why read on?}

- This is the first systematic analysis of SP-D functionality in any clinical population and highlights a novel functional deficiency in preterm infants of a molecule known to protect against inflammation and ventilator-induced injury in models of prematurity.

recognises carbohydrate structures on a wide spectrum of bacteria, fungi and viruses. ${ }^{4}$ Additionally, SP-D plays a critical role regulating inflammatory cell activity, ${ }^{5}$ and deficiency of this molecule results in a spontaneous and progressive inflammatory lung disease. ${ }^{6}$ SP-D exists in a variety of oligomeric states. Dodecamers, consisting of four trimeric subunits, form by covalent and non-covalent interactions between amino-terminal peptide sequences and these, along with trimers, are the most commonly isolated oligomers in humans. ${ }^{7}$ Larger oligomers, sometimes referred to as 'fuzzy balls' or 'astral bodies', are observed less frequently but have greater opsonic and antiviral activity. ${ }^{8}$ By contrast, naturally occurring trimers exhibit poor affinity for traditional carbohydrate ligands otherwise bound by corresponding dodecamers ${ }^{7}$

SP-D functional activity is influenced by a number of factors. One common polymorphism $\left(\mathrm{Thr}^{11}\right)$ substantially limits SP-D oligomerisation to trimers with restricted lectin activity. ${ }^{7}$ SP-D is also subject to structural and functional modification by elements of the innate inflammatory response, including neutrophilderived serine proteases, ${ }^{10}$ and oxidative mediators such as myeloperoxidase and peroxynitrite ${ }^{11} 12$ 
Recent gene association studies implicate variants of SP-D in the incidence of spontaneous preterm birth ${ }^{13}$ and the development of RDS in preterm infants. ${ }^{14}$ Low levels of SP-D are associated with infection in models of preterm ventilation ${ }^{15}$ and inflammation and hyperoxic stress in the mouse. ${ }^{16}{ }^{17}$ Exogenous SP-D therapy reduces endotoxic shock and ventilator-induced inflammation in preterm lambs. ${ }^{18}{ }^{19}$ Despite this, only one study has investigated SP-D expression in ventilated preterm infants at risk of CLD, describing lower levels in those who develop CLD. ${ }^{20}$ No studies have yet described the functional status of SP-D in this or any other clinical cohort. Here we examine the expression and lectin activity of SP-D in bronchoalveolar lavage fluid (BALF) from a cohort of ventilated preterm and term infants.

\section{MATERIALS AND METHODS}

\section{Patient recruitment and sample processing}

BALF was obtained from ventilated preterm and term infants recruited as part of a previously published study. ${ }^{21}$ Full details of sample collection and processing are provided in the online supplementary materials and methods. Ethical approval was obtained from the local Research Ethics Committee and written informed consent was obtained from the parents. Cell-free supernatants were stored at $-80^{\circ} \mathrm{C}$ within $30 \mathrm{~min}$ of collection. Prior to analysis, samples were thawed and subjected to microcentrifugation at $13000 \mathrm{rpm}$ for $1 \mathrm{~min}$. Not all samples from the original study were available for analysis, and for a small number of samples, sufficient volume was not available to enable analysis in ELISA and zymosan binding assays (see table 1; figures 1, 3 and 4, panels C,D). Data were excluded from two preterm infants with large sacrococcygeal teratomas.

\section{Reagents}

All reagents were from Fisher Scientific (Loughborough, UK) unless otherwise stated. Goat anti-SP-D was from R\&D systems (Abingdon, UK). Minimally cross-reactive horseradish peroxidase (HRPO)-conjugated donkey anti-goat IgG and donkey antirabbit IgG was from Jackson ImmunoResearch (Suffolk, UK). Native SP-D was purified as previously described ${ }^{22}$ and stored at $-80^{\circ} \mathrm{C}$.

\section{ELISA}

SP-D was quantified using a two monoclonal antibody sandwich ELISA from Hycult Biotech (Uden, The Netherlands) according to the manufacturer's instructions (measurable range $6.3-400 \mathrm{ng} / \mathrm{ml}$ ).
BALF samples were diluted between $1: 10$ and $1: 160$ and measured in duplicate.

\section{Zymosan and maltose-agarose binding assay}

Full methodology is detailed in the online supplementary materials and methods. Briefly, native SP-D $(2 \mu \mathrm{g} / \mathrm{ml}$ in $154 \mathrm{mM}$ $\mathrm{NaCl}$ ) or neonatal BALF was diluted $1: 1$ in tris-buffered saline (TBS), pH 7.6 with $10 \mathrm{mM} \mathrm{CaCl} 2$ or $10 \mathrm{mM}$ ethylenediaminetetraacetic acid (EDTA) or $100 \mathrm{mM}$ D-maltose. This was incubated with a washed pellet from $10 \mu \mathrm{l}$ of a $1 \% \mathrm{w} / \mathrm{v}$ suspension of Zymosan-A or $10 \mu \mathrm{l}$ maltose-agarose. If indicated, native SP-D was preincubated with an equal volume of BALF prior to substrate binding, which proceeded for $30 \mathrm{~min}$ at $37^{\circ} \mathrm{C}$. Following microcentrifugation, supernatants were carefully aspirated, the pellet was washed once and SP-D eluted with TBS-EDTA. In some experiments, supernatants were subjected to a further round of zymosan binding. In other experiments supernatants were subsequently assessed for mannan binding activity as described below. Samples for sodium dodecyl sulfate polyacrylamide gel electrophoresis (SDS-PAGE) were immediately boiled for 2 min with SDS sample buffer with or without $\beta$ mercaptoethanol.

\section{SDS-PAGE and western blot}

Full methodology is detailed in the online supplementary information. Briefly, samples were separated on 10\% SDS-PAGE gels, transferred to nitrocellulose and sequentially incubated with $0.1 \mu \mathrm{g} / \mathrm{ml}$ goat anti-human SP-D and HRPO-conjugated donkey anti-goat IgG in phosphate buffered saline (PBS) milk. Blots developed with ECL prime (GE Healthcare LifeSciences, Bucks, UK) were subjected to densitometry as previously described using ImageJ V.1.46. ${ }^{23}$ Images presented display all visible bands from developed blots.

\section{Solid phase binding assays}

Full methodology is detailed in the online supplementary information. Briefly, BALF pre-zymosan and post-zymosan binding, or native SP-D was diluted in TBS with $0.05 \%(\mathrm{v} / \mathrm{v})$ Tween (TBST), pH 7.6 plus $10 \mathrm{mM} \mathrm{CaCl}_{2}$ (TBST-C) as detailed in the results section. Some assays were carried out in the presence of $100 \mathrm{mM}$ D-maltose or $100 \mu \mathrm{g} / \mathrm{ml}$ Poractant Alfa (Chiesi, Cheadle, UK); others excluded calcium but included $10 \mathrm{mM}$ EDTA (TBST-E). Binding to mannan $(50 \mu \mathrm{g} / \mathrm{ml})$ or PBS-coated 96-well Nunc MaxiSorp plates proceeded for $90 \mathrm{~min}$ at room temperature. Wells were washed with TBST-C or TBST-E prior to incubation with goat anti-SP-D $(1 \mu \mathrm{g} / \mathrm{ml})$ in TBST-C or, in

Table 1 Patient characteristics

\begin{tabular}{llll}
\hline & $\begin{array}{l}\text { Preterm } \\
\text { CLD }\end{array}$ & $\begin{array}{l}\text { Preterm } \\
\text { No CLD }\end{array}$ \\
\hline Number of patients & 14 & 14 & 5 \\
Number of samples: (ELISA/functional assay) & $50(45 / 48)$ & $32(30 / 32)$ & $16(15 / 16)$ \\
Gestational age (weeks)* $\dagger$ & $26^{+2}\left(25^{+4}-29^{+1}\right)$ & $28^{+5}\left(27^{+1}-29^{+3}\right)$ & Term \\
Birth weight $(\mathrm{g}) \dagger$ & $960(850-1330)$ & $1140(977-1257)$ & $2710(2390-2730)$ \\
Prolonged rupture of membranes $(>24 \mathrm{~h})$ & $4 / 14(29 \%)$ & $1 / 14(7 \%)$ & $0 / 5(0 \%)$ \\
Antenatal steroids $(>24 \mathrm{~h})$ & $11 / 14(79 \%)$ & $10 / 14(71 \%)$ & $0 / 5(0 \%)$ \\
Surfactant & $14 / 14(100 \%)$ & $14 / 14(100 \%)$ & $0 / 5(0 \%)$ \\
Caesarean delivery & $7 / 14(50 \%)$ & $9 / 14(64 \%)$ & $1 / 5(20 \%)$ \\
\hline *Values in superscript refer to days in addition to weeks of gestation. & & & \\
tValues are reported as median (IQR). & & & \\
CLD, chronic lung disease. & &
\end{tabular}



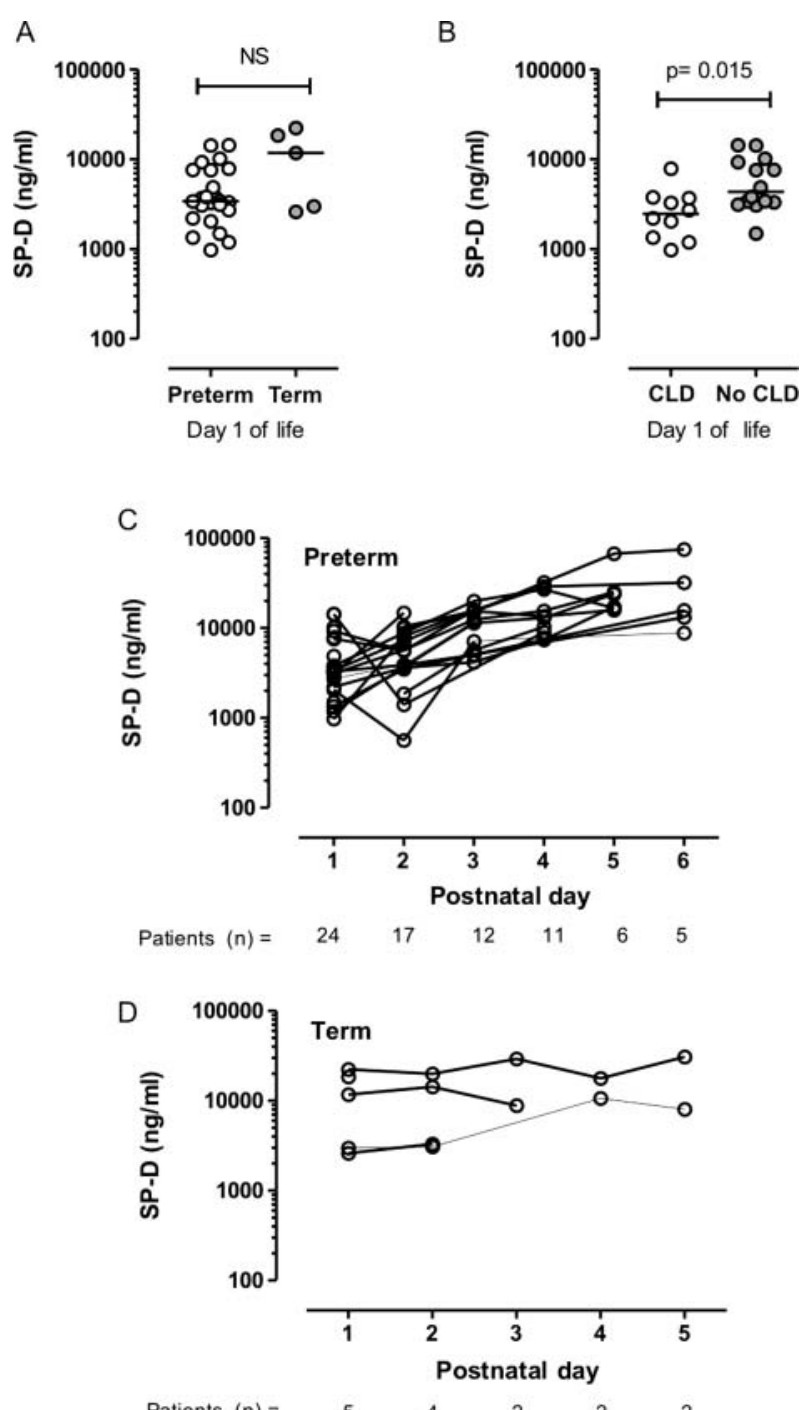

Figure 1 Surfactant protein D (SP-D) expression in neonatal bronchoalveolar lavage fluid (BALF): SP-D was quantified by ELISA in the indicated number of BALF samples from 28 preterm and five term infants. (A) SP-D concentration on day 1 of life in preterm $(n=24)$ and term infants $(n=5)$. (B) SP-D concentration on day 1 of life was lower in preterm infants who developed chronic lung disease $(n=10)$ than those who did not $(n=14)$. (C and D) SP-D concentration was plotted against the day of life for preterm and term infants. In all cases data points for SP-D are the average of duplicate measurements. Note that SP-D concentration is plotted on a logarithmic axis. CLD, chronic lung disease.

the case of assays with Poractant Alfa, rabbit anti-SP-D $(5 \mu \mathrm{g} / \mathrm{ml})$ in TBST-C for $1 \mathrm{~h}$ at room temperature. Wells were washed three times with TBST-C prior to incubation with HRPO-conjugated donkey anti-goat IgG or donkey anti-rabbit IgG in TBST-C for $30 \mathrm{~min}$ at room temperature. Wells were washed with TBST-C, developed with TMB substrate (eBioscience, Hatfield, UK) and quenched with $1 \mathrm{M} \mathrm{H}_{2} \mathrm{SO}_{4}$ prior to reading at $450 \mathrm{~nm}$ on a MRX TC Revelation plate reader (Dynex Technologies, West Sussex, UK).

\section{Statistical analysis}

All data are expressed as medians and IQRs unless otherwise stated. Statistical analysis was performed with GraphPad Prism V.5.01. Differences in the medians of continuous data were analysed by Mann-Whitney $U$ test. In all cases, significance was achieved at $\mathrm{p}$ values $<0.05$.

\section{RESULTS}

Expression of SP-D in BALF from term and preterm ventilated infants

Full patient demographics are described in table 1. SP-D expression in term BALF on day 1 of life $(11730 \mathrm{ng} / \mathrm{ml} ; 2788-$ $20340)$ was greater than in preterm BALF $(3401 \mathrm{ng} / \mathrm{ml} ; 2330$ 7582), but not significantly so (figure 1A). SP-D expression was significantly lower on day 1 of life in preterm infants who developed CLD (2460 ng/ml; 1301-3696) compared with those who did not $(4334 \mathrm{ng} / \mathrm{ml} ; 3265-9478 ; \mathrm{p}=0.015)$ (figure 1B). Expression increased over the first 6 days of life in the majority of infants, particularly in the preterm cohort (figures 1C,D).

\section{An assay to measure the lectin activity of SP-D}

The ability of native purified SP-D to interact with zymosan and migrate to a pellet with these particles under centrifugation was tested. Following SDS-PAGE and western blot of pellet and supernatant fractions under reducing conditions a band of approximately $48 \mathrm{kDa}$, corresponding to monomeric subunits of SP-D, mobilised to the zymosan pellet in the presence $\mathrm{Ca}^{2+}$ (figure 2A and see online supplementary figure S1). The highest concentration of SP-D tested in preliminary experiments was $20 \mu \mathrm{g} / \mathrm{ml}$ (data not shown). The majority of SP-D remained in the supernatant fraction in the presence of EDTA and maltose, confirming the calcium and carbohydrate dependence of the interaction (figure 2A and see online supplementary figure S1).

\section{A large proportion of BALF SP-D from term and preterm infants is incapable of binding to zymosan}

Zymosan binding activity was tested in 80 BALF samples from 28 preterm infants and 16 BALF samples from five term infants. In addition to zymosan-bound SP-D, a band of identical molecular weight was observed in supernatant fractions from all BALF samples tested (figure $2 \mathrm{~B}$ ). A minor band approximately $2 \mathrm{kDa}$ below the main band was frequently observed in pellet and supernatant fractions (figure $2 \mathrm{~B}$ ) while a band mobilising $4-5 \mathrm{kDa}$ higher than the main SP-D monomer was often observed, but only in supernatant fractions (figure 2B). Sedimentation of SP-D did not occur independently of zymosan interactions which were calcium and carbohydrate dependent (figure 2C). Supernatant fractions containing non-bound SP-D did not exhibit substantial additional binding in a second round of zymosan binding, ruling out the possibility that ligand binding sites on the initial zymosan particles had been saturated (see online supplementary figure S2).

\section{Therapeutic surfactant or other factors intrinsic to BALF do} not inhibit SP-D zymosan binding activity

Pulmonary surfactant, previously reported to interact with SP-D, ${ }^{24}$ had no effect on SP-D binding to mannan in a solid phase assay (see online supplementary figure S3). Furthermore, BALF samples in which all or most of the SP-D was found in the supernatant fraction were unable to inhibit exogenously spiked native SP-D binding to zymosan (figure 2D). These data suggest that an intrinsic inhibitor of SP-D binding within such BALF samples was not responsible for the lack of zymosan binding activity.

\section{SP-D binding activity in term and preterm infants increases} over the first week of life

Zymosan binding assays undertaken for all term and preterm BALF samples collected over the first 6 days of life were 

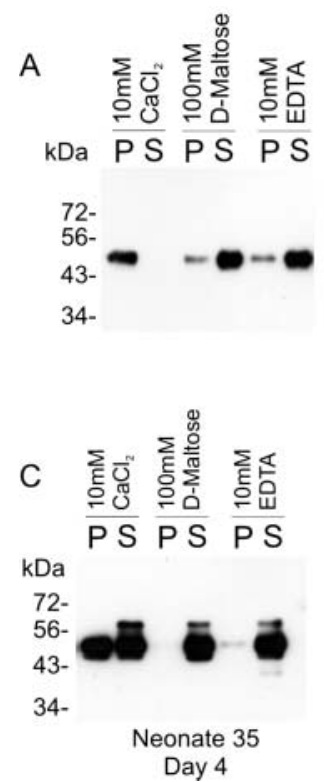
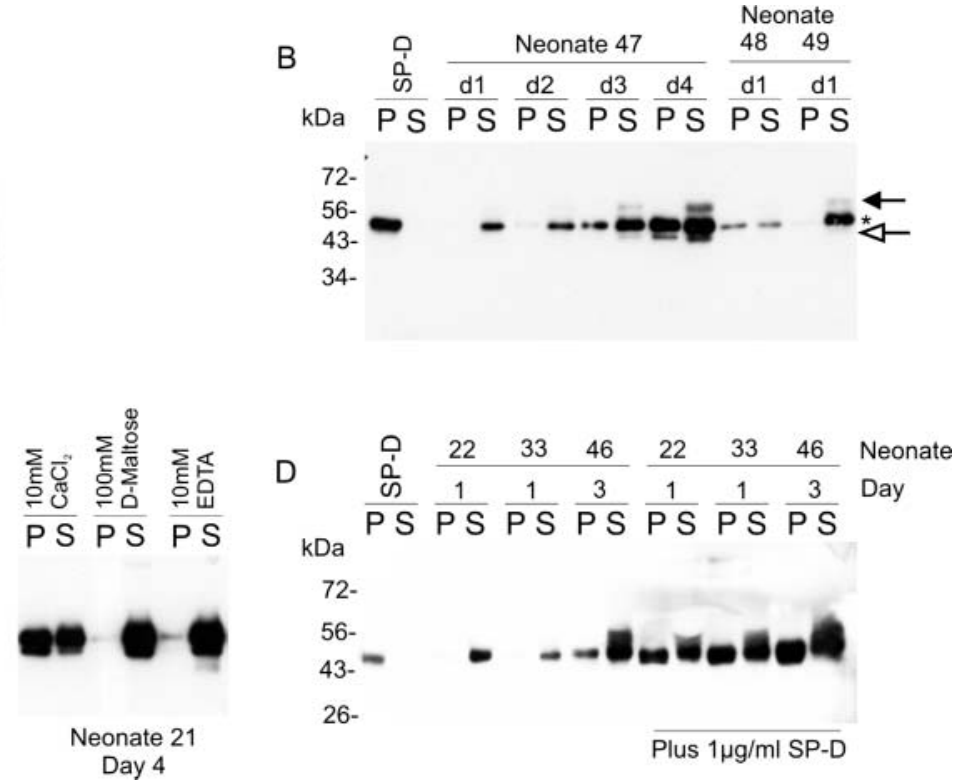

Figure 2 An assay for surfactant protein D (SP-D) lectin activity reveals significant amounts of SP-D in neonatal bronchoalveolar lavage fluid (BALF) which does not bind to zymosan. (A) Western blot of pellet (P) and supernatant (S) fractions from zymosan binding assays reveals binding of native SP-D to zymosan is inhibited by calcium chelation with ethylenediaminetetraacetic acid (EDTA) and by 100 mM D-maltose. A major band migrating between 43 and $56 \mathrm{kDa}$ corresponds to the expected molecular weight for fully reduced SP-D monomeric subunits. (B) Native SP-D or BALF from preterm infants was added to a washed zymosan pellet in the presence of $10 \mathrm{mM} \mathrm{CaCl}$. Bound (P) and non-bound (S) SP-D was visualised by western blot of respective fractions. In the native SP-D positive control, a major band migrating between 43 and $56 \mathrm{kDa}$ (asterix) was observed only in the pellet fraction. An equivalent major band was observed in pellet and supernatant fractions from BALF. In some BALF samples all SP-D reactive material was present in the non-bound supernatant fraction (neonate 47, day 1 and 2; neonate 49, day 1). In others this band was present in bound pellet fractions and non-bound supernatant fractions. An additional band migrating 3-5 kDa above the major SP-D reduced monomer (closed arrow) was frequently observed, but exclusively in supernatant fractions (neonate 47, day 3 and 4; neonate 49, day 1). A further band migrating 1-2 kDa below the main SP-D band (open arrow) was also frequently observed but was present in supernatant and pellet fractions (neonate 47, day 3 and 4; neonate 49, day 1). Data are representative of all bands visible on duplicate blots from at least 21 independent experiments. (C) SP-D from neonatal BALF binds to zymosan in a D-maltose and EDTA inhibitable fashion. Blots represent two of three independent experiments. (D) Neonatal BALF containing SP-D which was predominantly unable to interact with zymosan (lanes 3-8) was spiked with native SP-D $(1 \mu \mathrm{g} / \mathrm{ml})$ for $30 \mathrm{~min}$ at $37^{\circ} \mathrm{C}$, before adding it to a freshly washed zymosan pellet. The exogenous SP-D retained its capacity to interact with zymosan in the presence of BALF from neonates with endogenous SP-D which was unable to interact with zymosan (lanes 9-14). Data are representative of three independent experiments.

performed in duplicate to confirm reproducibility (representative western blots are presented in online supplementary figure S4AD). Densitometry following assay of all available samples revealed the percentage of SP-D capable of binding to zymosan to be more than three times higher in term infants on day 1 of life $(47.85 \%$; 21.39-48.51) compared with preterm infants $(13.35 \% ; 2.03-28.13)$ (figure 3A). Close to $100 \%$ of positive control native SP-D mobilised to the pellet in these assays (data not shown). Binding activity on day 1 of life did not differ between infants who developed CLD and those who did not (figure 3B). A pattern of increased SP-D binding activity was evident in preterm BALF over the first 6 days of life, peaking on day 5 (median 50.76\%, IQR 29.18-55.40) (figure 3C). A less obvious pattern punctuated by spikes in activity was noted in the term infants followed over the first 5 days of life (figure 3D).

Combining these data with those from the ELISA, the amount of SP-D capable of binding to zymosan was seen to increase substantially over the first 5 days of life, particularly in preterm infants (figure 4C,D). The concentration of SP-D with binding activity was almost 10 times lower in preterm infants $(373 \mathrm{ng} / \mathrm{ml} ; 83-1537)$ than term infants $(3668 \mathrm{ng} / \mathrm{ml} ; 1030$ $7210)$ on day 1 of life $(p=0.014)$ (figure $4 A$ ). Substantial (but not significant; $\mathrm{p}=0.12$ ) differences in SP-D binding zymosan were observed between those who developed CLD $(237 \mathrm{ng} / \mathrm{ml}$; 27-399) and those who did not (1105 ng/ml; 143.5-1990) (figure 4B).

\section{A large proportion of SP-D from preterm BALF also fails to bind to maltose-agarose}

Maltose-agarose, a well described affinity matrix for SP-D, did not support the binding of a significant proportion of SP-D in BALF and in some cases supported the binding of less SP-D than zymosan binding assays carried out in parallel (see online supplementary figure S5). As observed in zymosan binding assays, an additional band mobilising $4-5 \mathrm{kDa}$ higher than the main SP-D monomer was also observed in non-bound supernatant fractions (see online supplementary figure S5). A minor band migrating $1-2 \mathrm{kDa}$ below the major band was also observed in pellet and supernatant fractions from both assays (see online supplementary figure S5).

\section{SP-D which fails to bind zymosan exhibits characteristics of sub-dodecameric oligomeric form}

SP-D in supernatant and pellet fractions from zymosan binding assays was examined by SDS-PAGE under non-reducing conditions to assess differences in oligomeric form (figure 5A). The majority of zymosan-bound SP-D was characterised by highly oligomerised forms too large to enter the running gel (figure $5 B)$. By contrast, SP-D which did not bind to zymosan migrated with the predicted mass of trimers and also included apparent dimeric and monomeric material, indicating the presence of lower oligomeric forms (figure 5B). Monomeric material was also occasionally observed in the bound, pellet fraction. 


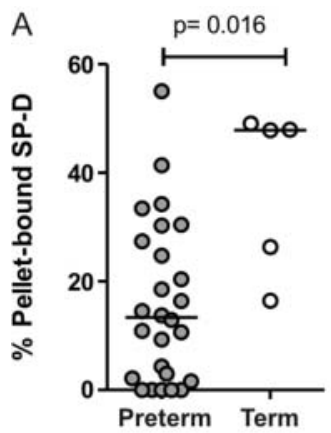

Day 1 of life

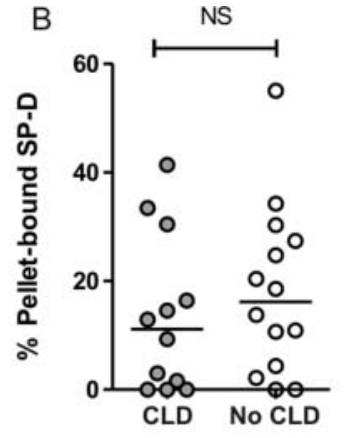

Day 1 of life
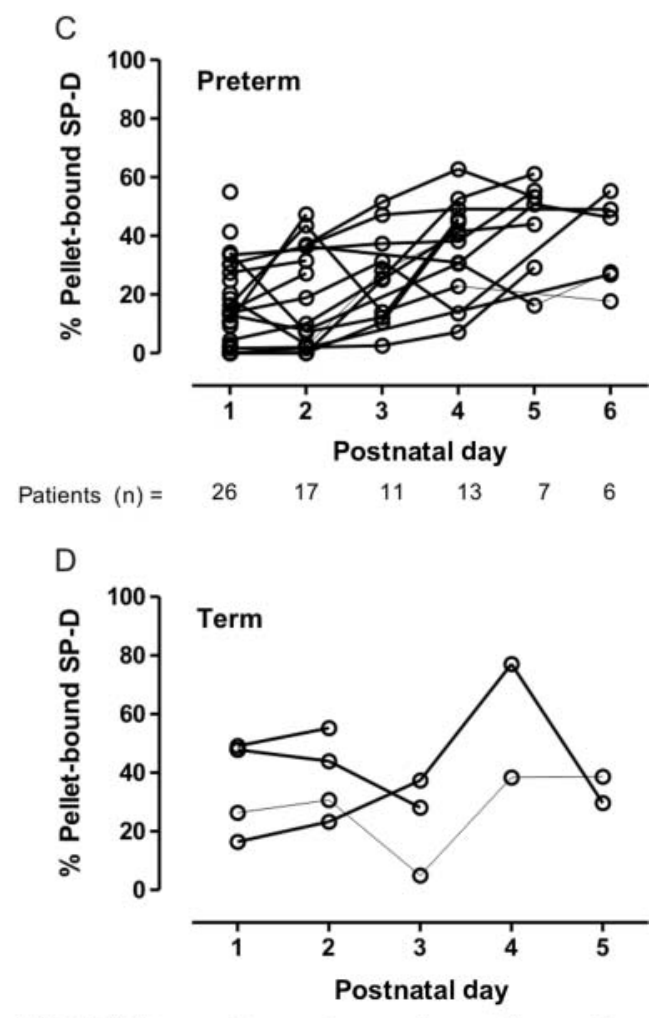

Patients $(n)=\begin{array}{lllll}5 & 4 & 3 & 2 & 2\end{array}$

Figure 3 Analysis of surfactant protein D (SP-D) binding activity in all neonatal bronchoalveolar lavage fluid (BALF). Two independent zymosan binding assays were performed on each BALF sample from 28 preterm infants and five term infants. Native SP-D was used as a positive control in all assays. SP-D in bound pellet and non-bound supernatant fractions was visualised following sodium dodecyl sulfate polyacrylamide gel electrophoresis under reducing conditions and western blot. Individual western blots for each experiment were subjected to densitometry to quantify the relative proportions of bound and non-bound SP-D. Data presented refer to the percentage of total SP-D density associated with the pellet-bound fraction. (A and B) The percentage of zymosan bound SP-D was significantly higher in BALF from term infants on the first day of life $(n=5)$ compared with preterm infants $(n=26, p=0.016)$ but did not differ between infants who developed chronic lung disease $(n=12)$ and those who did not $(n=14)$. Bars refer to medians. ( $C$ and D) The percentage of pellet-bound SP-D for the indicated number of BALF samples was plotted against postnatal day for preterm and term infants.

\section{SP-D which fails to bind zymosan is also unable to bind mannan}

SP-D in supernatant fractions from zymosan binding assays was tested in plate-based mannan binding assays. BALF with approximately $50 \%$ of SP-D capable of interacting with
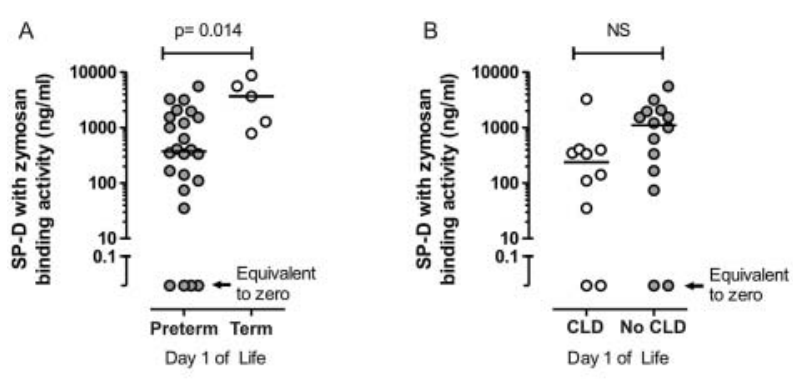

C

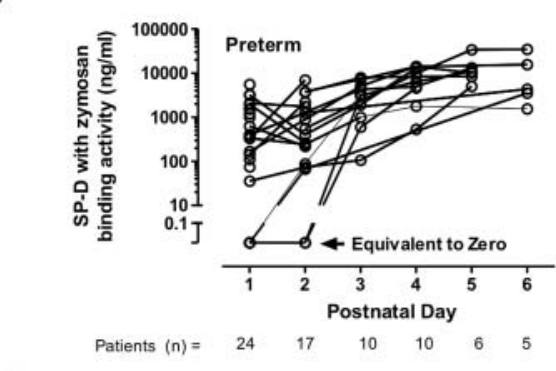

D

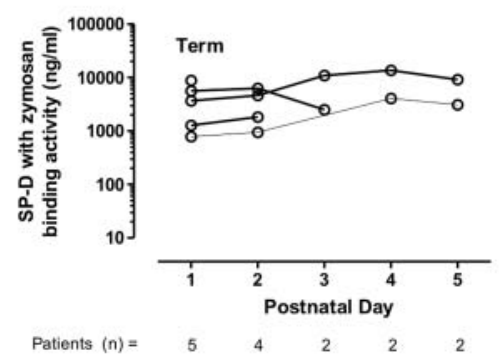

Figure 4 Total surfactant protein D (SP-D) with zymosan binding activity is higher in term than preterm neonatal bronchoalveolar lavage fluid (BALF). Data from ELISA presented in figure 1 and the zymosan binding assay described in figure 3 were used to calculate the amount of SP-D in BALF with zymosan binding activity. (A) Term neonatal BALF $(n=5)$ contained significantly more SP-D with the capacity to bind zymosan on day 1 of life compared with preterm neonatal BALF ( $n=24$, $p=0.014)$. (B) The concentration of SP-D with zymosan binding activity in BALF from infants who subsequently developed chronic lung disease $(n=10)$ was not significantly different from those who did not $(n=14$, $p=0.12$ ). (C) Preterm neonates exhibited a pattern of increasing concentrations of SP-D capable of binding to zymosan over the first 6 days of life. (D) The amount of SP-D capable of binding to zymosan in BALF from term neonates remained relatively stable across the first 5 days of life.

zymosan was selected for these assays (zymosan binding assays run in parallel are shown in online supplementary figure S6B). Following zymosan binding, supernatant fractions were adjusted to achieve $1 \mu \mathrm{g} / \mathrm{ml} \mathrm{SP-D} \mathrm{(ELISA} \mathrm{equivalent)} \mathrm{and} \mathrm{this} \mathrm{was} \mathrm{used} \mathrm{as}$ source material for binding assays. Also included was nonassayed BALF adjusted to $2 \mu \mathrm{g} / \mathrm{ml}$ total SP-D (giving the equivalent of $1 \mu \mathrm{g} / \mathrm{ml}$ of material capable of binding to zymosan and $1 \mu \mathrm{g} / \mathrm{ml}$ of non-binding material). Native SP-D was used as a positive control. In all cases, post-zymosan supernatant fractions exhibited negligible binding to mannan (see online supplementary figure S6A). By contrast, SP-D from equivalent non-assayed BALF exhibited EDTA and maltose inhibitable binding to mannan comparable to native SP-D.

\section{DISCUSSION}

We have presented the first systematic evaluation of SP-D functionality in any clinical cohort. Our data reveal substantial and 

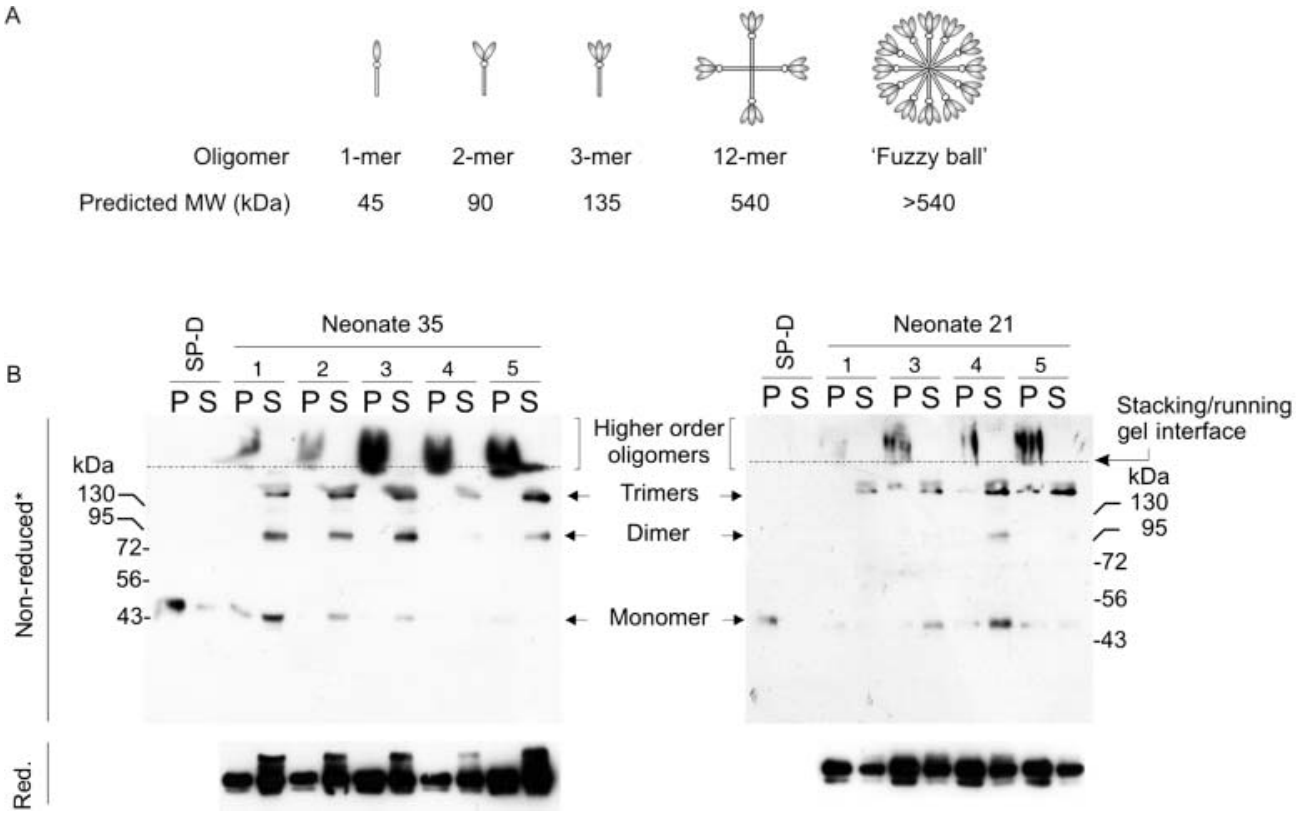

Figure 5 Surfactant protein D (SP-D) which fails to bind zymosan is of a lower oligomeric state than bound SP-D. (A) Schematic representation of oligomeric variants of SP-D and their approximate predicted molecular weights (MWs). These MWs can differ from those observed on sodium dodecyl sulfate polyacrylamide gel electrophoresis (SDS-PAGE) due to differences in post-translational modification, buffer system and molecular weight standards used. (B) A series of bronchoalveolar lavage fluids from two different neonates were assessed in a zymosan binding assay and pellet-bound and supernatant non-bound fractions were subsequently separated by SDS-PAGE under non-reducing (top panels) or reducing (bottom panels) conditions, followed by western blot for SP-D. * The native SP-D positive control was only run under reducing conditions and is presented in the upper panels. Dashed lines on non-reduced gels indicate the approximate position of the interface between the $4 \%$ stacking gel and $10 \%$ running gel. Under non-reducing conditions SP-D eluted from the zymosan pellet appears predominantly either as large oligomers which fail to enter the running gel (neonate 35) or as a combination of these large oligomers and forms migrating with a MW indicative of trimers (neonate 21). This denatured banding pattern indicates the presence of dodecameric and larger forms. By contrast all material in the non-bound supernatant fractions consisted of bands consistent with trimeric, dimeric and monomeric forms of SP-D, indicative of lower oligomeric forms. Occasionally, monomeric forms were also visible in the zymosan-bound pellet fraction (neonate 21, days 4 and 5). Data for non-reducing conditions are representative of three independent experiments.

significant reduction of SP-D expression in preterm infants who develop CLD and also identifies a significant restriction of SP-D lectin activity in preterm infants on day 1 of life related to the oligomeric state of that molecule.

BALF was collected from preterm and term infants undergoing mechanical ventilation as previously reported, ${ }^{21}$ and according to published guidelines. ${ }^{25}$ Although the cohort under investigation was relatively small, the reduced SP-D expression in preterm infants who develop CLD and the increased SP-D expression over the first 6 days of life broadly agree with data from the only previous study in a similar population. ${ }^{20}$ Although SP-D expression varied considerably in the limited number of term infants available to us, a pronounced increase in expression over the first 5 days was not observed. Increased BALF SP-A and SP-B expression over the first week of life has also been reported in a preterm population, ${ }^{20}$ suggestive of increased secretory protein expression as the lung matures. In addition to being more gestationally mature, only term neonates exposed to $\leq 28 \% \mathrm{O}_{2}$ during respiratory support were included here and so were subject to milder ventilation than the preterm cohort. Lung maturation over the first week of life combined with exposure to mechanical ventilation may explain some of the increased SP-D expression in the preterm population, however the limited number of term infants available demands a cautious interpretation until a larger cohort enables a more detailed analysis. SP-D expression in lung tissue increases in late gestation and is glucocorticoid responsive in humans ${ }^{26}$ and rodents. ${ }^{27}$ Here, SP-D expression in preterm infants on day 1 of life did not correlate with gestational age and did not differ depending on mode of delivery or in those born to mothers who received antenatal steroids $(>24 \mathrm{~h}$ ) (data not shown). Furthermore, although interactions between SP-D and inflammatory cells have been reported, ${ }^{5} 28$ statistically significant differences in total cell, neutrophil or macrophage numbers were not observed between infants who developed CLD and those who did not on day 1 (data not shown), suggesting that separation of cells and supernatant during BALF processing does not explain differences in SP-D expression between groups, a possibility experimentally ruled out in other inflammatory lung diseases. $^{29}$

In addition to this quantitative deficiency, a significant proportion of term and preterm BALF SP-D failed to bind the Saccharomyces cerevisiae derived particle, zymosan. Zymosan saturation or a competing factor within the BALF did not appear to explain the functional defect and the SDS-PAGE migration pattern did not suggest proteolytic degradation, previously reported to alter SP-D activity. ${ }^{10}$ However, the frequent presence of an apparent isoform of SP-D migrating $4-5 \mathrm{kDa}$ above the main monomeric subunit was reminiscent of a differentially glycosylated $50 \mathrm{kDa}$ form of SP-D described by Mason et $a l^{30}$ and thought to limit SP-D oligomerisation to trimers. Furthermore, SP-D which failed to bind zymosan migrated on SDS-PAGE with a pattern previously reported for low oligomeric forms of SP-D, ${ }^{7}$ consisting predominantly of subdodecameric species. Conversely, zymosan-bound SP-D migrated with a pattern described for dodecameric and highly oligomerised variants of SP-D. ${ }^{7}$ Restricted oligomerisation of SP-D 
limits its ability to interact with a range of pathogens ${ }^{78}$ and purified carbohydrate ligands, ${ }^{7} 9$ however this is the first time this phenomenon has been systematically addressed in a relevant clinical population. The restricted binding activity of SP-D from term and preterm BALF towards maltose-agarose and mannan is also likely to represent lower oligomeric forms as previously reported. $^{79}$

In addition to its lectin activity, SP-D exhibits additional activity in regulating inflammatory processes, most clearly evident in the emphysematous, inflammatory lung disease observed in the SP-D knockout mouse. ${ }^{6} 31$ The mechanism underlying this phenotype is not fully understood but is thought to involve interactions between SP-D and inflammatory cells either to regulate their activity $^{53}$ or to promote clearance of apoptotic bodies during inflammatory resolution. ${ }^{28}{ }^{33}$ Genetic reconstitution of knockout mice with a version of SP-D lacking residues required for full oligomerisation failed to rescue the emphysematous phenotype, ${ }^{34}$ implying a critical role for full oligomerisation in pulmonary homeostasis. However, recombinant trimeric fragments of SP-D with a minimal collagenous tail region also exhibit significant immunomodulatory activity in vivo $^{28} 35$

We identified substantial variation in the status of SP-D in preterm infants at risk of CLD. Existing therapeutic surfactant used in this population lacks SP-D. Replacement therapy has shown promise in the treatment of endotoxic shock and ventilator-induced inflammation in preterm lambs, ${ }^{18} 19$ and pulmonary overexpression of SP-D protects mice from acute hyperoxic lung injury. ${ }^{17}$ In this context a quantitative and functional deficiency of SP-D in the preterm population may limit their ability to appropriately regulate pulmonary inflammation, a key factor in the development of CLD. The importance of oligomeric size in mediating the anti-inflammatory effects of SP-D remains unclear but should be further investigated before considering the best approach to SP-D therapy in this population.

Acknowledgements We are grateful to Dr Martha Triantafilou for critical reading of the manuscript.

Contributors We confirm that all named authors made a significant contribution to each of the following areas: conception and design, or analysis and interpretation of data; drafting the article or revising it critically for important intellectual content; final approval of the version to be published. We also confirm that no person who fulfils these criteria has been omitted as an author. Eamon McGreal conceived and designed the experimental aspects of the study, undertook experimental work, analysed the data and drafted the manuscript. He acts as guarantor. Philip Davies analysed and interpreted clinical aspects of the study, revised the article for intellectual content and gave final approval of the version to be published. Howard Clark collaborated in the conception and design of experimental aspects of the study, revised the article for intellectual content and gave final approval of the version to be published. Sailesh Kotecha conceived and designed clinical aspects of the study, was involved in analysis and interpretation of the data, revised the article for intellectual content and gave final approval of the version to be published.

Funding Dr Phil Davies was supported by funding from Arriva Pharmaceuticals Inc; CYPRN supported a Research Development Group led by Eamon McGreal to support the development of an innate immune research group in the neonatal population. These funders were not involved in data collection, analysis or interpretation, and they were not involved in the preparation or final approval of the manuscript.

Competing interests None.

Ethics approval South East Wales Research Ethics Committee.

Provenance and peer review Not commissioned; externally peer reviewed.

\section{REFERENCES}

1 Levy 0. Innate immunity of the newborn: basic mechanisms and clinical correlates. Nat Rev Immunol 2007;7:379-90.

2 McGreal EP, Hearne K, Spiller OB. Off to a slow start: under-development of the complement system in term newborns is more substantial following premature birth. Immunobiology 2012;217:176-86.
3 Chakraborty M, McGreal EP, Kotecha S. Acute lung injury in preterm newborn infants: mechanisms and management. Paediatr Respir Rev 2010;11:162-70.

4 Whitsett JA. Surfactant proteins in innate host defense of the lung. Biol Neonate 2005;88:175-80

5 Gardai SJ, Xiao YQ, Dickinson M, et al. By binding SIRPalpha or calreticulin/CD91, lung collectins act as dual function surveillance molecules to suppress or enhance inflammation. Cell 2003:115:13-23.

6 Wert SE, Yoshida M, LeVine AM, et al. Increased metalloproteinase activity, oxidant production, and emphysema in surfactant protein $D$ gene-inactivated mice. Proc Natl Acad Sci U S A 2000;97:5972-7.

7 Leth-Larsen R, Garred P, Jensenius H, et al. A common polymorphism in the SFTPD gene influences assembly, function, and concentration of surfactant protein D. J Immunol 2005;174:1532-8.

8 Hartshorn $\mathrm{K}$, Chang D, Rust K, et al. Interactions of recombinant human pulmonary surfactant protein D and SP-D multimers with influenza A. Am J Physiol 1996;271 (5 Pt 1):L753-62.

9 Crouch EC, Smith K, McDonald B, et al. Species differences in the carbohydrate binding preferences of surfactant protein D. Am J Respir Cell Mol Biol 2006;35:84-94

10 Griese $M$, Wiesener $A$, Lottspeich $F$, et al. Limited proteolysis of surfactant protein D causes a loss of its calcium-dependent lectin functions. Biochim Biophys Acta 2003;1638:157-63.

11 Matalon S, Shrestha K, Kirk M, et al. Modification of surfactant protein D by reactive oxygen-nitrogen intermediates is accompanied by loss of aggregating activity, in vitro and in vivo. FASEB J 2009;23:1415-30.

12 Crouch EC, Hirche TO, Shao B, et al. Myeloperoxidase-dependent inactivation of surfactant protein D in vitro and in vivo. J Biol Chem 2010;285:16757-70.

13 Karjalainen MK, Huusko JM, Tuohimaa A, et al. A study of collectin genes in spontaneous preterm birth reveals an association with a common surfactant protein D gene polymorphism. Pediatr Res 2012;71:93-9.

14 Hilgendorff $A$, Heidinger $K$, Bohnert $A$, et al. Association of polymorphisms in the human surfactant protein-D (SFTPD) gene and postnatal pulmonary adaptation in the preterm infant. Acta Paediatr 2009;98:112-17.

15 Awasthi S, Coalson JJ, Yoder BA, et al. Deficiencies in lung surfactant proteins A and $D$ are associated with lung infection in very premature neonatal baboons. $A m J$ Respir Crit Care Med 2001;163:389-97.

16 Jain $\mathrm{D}$, Atochina-Vasserman E, Kadire $\mathrm{H}$, et al. SP-D-deficient mice are resistant to hyperoxia. Am J Physiol Lung Cell Mol Physiol 2007;292:L861-71.

17 Jain D, Atochina-Vasserman EN, Tomer Y, et al. Surfactant protein D protects against acute hyperoxic lung injury. Am J Respir Crit Care Med 2008;178:805-13.

18 Ikegami M, Carter K, Bishop K, et al. Intratracheal recombinant surfactant protein D prevents endotoxin shock in the newborn preterm lamb. Am J Respir Crit Care Med 2006:173:1342-7.

19 Sato A, Whitsett JA, Scheule RK, et al. Surfactant protein-D inhibits lung inflammation caused by ventilation in premature newborn lambs. Am J Respir Crit Care Med 2010;181:1098-105.

20 Beresford MW, Shaw NJ. Bronchoalveolar lavage surfactant protein A, B, and D concentrations in preterm infants ventilated for respiratory distress syndrome receiving natural and synthetic surfactants. Pediatr Res 2003;53:663-70.

21 Davies PL, Spiller OB, Beeton ML, et al. Relationship of proteinases and proteinase inhibitors with microbial presence in chronic lung disease of prematurity. Thorax 2010;65:246-51.

22 Strong P, Kishore U, Morgan C, et al. A novel method of purifying lung surfactant proteins $A$ and $D$ from the lung lavage of alveolar proteinosis patients and from pooled amniotic fluid. J Immunol Methods 1998;220:139-49.

23 McGreal EP, Davies PL, Powell W, et al. Inactivation of IL-6 and soluble IL-6 receptor by neutrophil derived serine proteases in cystic fibrosis. Biochim Biophys Acta 2010;1802:649-58

24 Ikegami M, Grant S, Korfhagen T, et al. Surfactant protein-D regulates the postnatal maturation of pulmonary surfactant lipid pool sizes. J Appl Physiol 2009:106:1545-2.

25 de Blic J, Midulla F, Barbato A, et al. Bronchoalveolar lavage in children. ERS Task Force on Bronchoalveolar Lavage in Children. European Respiratory Society. Eur Respir J 2000;15:217-31.

26 Dulkerian SJ, Gonzales LW, Ning Y, et al. Regulation of surfactant protein D in human fetal lung. Am J Respir Cell Mol Biol 1996;15:781-6.

27 Mariencheck W, Crouch E. Modulation of surfactant protein D expression by glucocorticoids in fetal rat lung. Am J Respir Cell Mol Biol 1994;10:419-29.

28 Clark H, Palaniyar N, Strong $P$, et al. Surfactant protein D reduces alveolar macrophage apoptosis in vivo. J Immunol 2002;169:2892-9.

29 Noah TL, Murphy PC, Alink JJ, et al. Bronchoalveolar lavage fluid surfactant protein-A and surfactant protein-D are inversely related to inflammation in early cystic fibrosis. Am J Respir Crit Care Med 2003;168:685-91.

30 Mason RJ, Nielsen LD, Kuroki Y, et al. A 50-kDa variant form of human surfactant protein D. Eur Respir J 1998;12:1147-55.

31 Botas C, Poulain F, Akiyama J, et al. Altered surfactant homeostasis and alveolar type II cell morphology in mice lacking surfactant protein D. Proc Natl Acad Sci U S A 1998:95:11869-74. 
32 Cai GZ, Griffin GL, Senior RM, et al. Recombinant SP-D carbohydrate recognition domain is a chemoattractant for human neutrophils. Am J Physiol 1999;276(1 Pt 1):L131-6.

33 Vandivier RW, Ogden CA, Fadok VA, et al. Role of surfactant proteins A, D, and C1q in the clearance of apoptotic cells in vivo and in vitro: calreticulin and CD91 as a common collectin receptor complex. J Immunol 2002;169:3978-86.
34 Zhang L, Ikegami M, Crouch EC, et al. Activity of pulmonary surfactant protein-D (SP-D) in vivo is dependent on oligomeric structure. J Biol Chem 2001:276:19214-19.

35 Knudsen L, Ochs M, Mackay R, et al. Truncated recombinant human SP-D attenuates emphysema and type II cell changes in SP-D deficient mice. Respir Res 2007;8:70. 